\title{
Conhecimentos, atitudes e comportamentos de estudantes Portugueses do ensino secundário relacionados com a prevenção da COVID-19
}

\author{
Regina Ferreira Alves ${ }^{1}$, Catarina Samorinha ${ }^{2}$, \& José Precioso ${ }^{1}$ \\ ${ }^{1}$ CIEC - Research Centre Child Studies, Institute of Education - University of Minho \\ 2 Sharjah Institute for Medical Research, University of Sharjah, Sharjah, United Arab Emirates
}

\begin{abstract}
Resumo: Este estudo pretendeu avaliar conhecimentos, atitudes e comportamentos acerca da COVID-19 em estudantes do Ensino Secundário em Portugal, considerando a sua importância para a adesão a medidas de prevenção desta doença. Estudantes do Ensino Secundário de todas as regiões do país $(\mathrm{N}=1258)$ preencheram um questionário online em maio de 2020. Os/as estudantes revelaram bons conhecimentos sobre a COVID-19, respondendo corretamente, em média, a 12 dos 14 itens, e reportaram atitudes maioritariamente positivas face aos comportamentos preventivos. Contudo, quanto à adoção destes comportamentos, em média, apenas 6 dos 12 comportamentos preventivos foram adotados. Ser do sexo feminino, frequentar cursos de Artes Visuais ou de Línguas e Humanidades, e mais atitudes positivas em relação à prevenção da COVID-19 aumentam as probabilidades de adotar comportamentos preventivos. Apresentam-se diferenças por sexo, ano de escolaridade, região e curso. Assim, é fundamental investir em campanhas preventivas que potenciem a adesão a comportamentos preventivos nesta população.
\end{abstract}

\section{Palavras-chave: COVID-19; estudantes; conhecimentos; atitudes, comportamentos preventivos.}

Knowledge, attitudes and behaviours of Portuguese students of secondary school related to the prevention of COVID-19: This cross-sectional study aimed to assess knowledge, attitudes and behaviours about COVID-19 among high school students in Portugal, given its importance for adherence to measures aimed at preventing this disease. High school students from all regions of the country $(\mathrm{N}=$ 1258) completed an online questionnaire in May 2020. Students revealed good knowledge about COVID19 , correctly answering 12 out of 14 items, and reported mostly positive attitudes towards preventive behaviours. However, the adoption of these behaviours was lower: only 6 out of 12 behaviours were adopted, on average, by students. Differences by sex, school year, region and course are presented. Being a female, attending courses on Visual Arts or Languages and Humanities, and having more positive attitudes towards prevention of COVID-19 increase the likelihood of adopting preventive behaviours. The investment in preventive campaigns is essential to enhance adherence to preventive behaviours in this population.

Keywords: COVID-19; students; knowledge; attitudes; preventive behaviours.

COVID-19 é a designação da Organização Mundial da Saúde (OMS) para a doença provocada pelo novo coronavírus, que pode causar infeção respiratória grave, como a pneumonia. As principais vias de transmissão da COVID-19, conhecidas à data da escrita deste manuscrito, são: 1) contacto próximo com pessoas infetadas pelo vírus ou 2) contacto com superfícies ou objetos contaminados. A transmissão acontece, assim, através de gotículas que se emitem, por exemplo, quando se tosse ou espirra, ou através do contacto de mãos contaminadas que, posteriormente, contactam os olhos, nariz ou a boca (as mãos contaminam-se facilmente em contacto com objetos ou superfícies por sua vez contaminadas com gotículas de pessoa infetada) (SNS, 2020).

Trata-se de uma doença contagiosa muito prevalente, a nível nacional e internacional, registandose à data de hoje (13 de setembro de 2020) 28637952 casos diagnosticados mundialmente (WHO, 2020a). Em Portugal, o registo dos primeiros casos confirmados de infeção pelo novo coronavírus ocorreu a 3 de março de 2020 (DGS, 2020a) e contabilizam-se atualmente 65021 casos confirmados e 1875 óbitos (DGS, 2020b). A COVID-19 está a ter reflexos negativos a nível individual, familiar, na sociedade e na economia, de uma dimensão sem precedentes. 0 risco de contágio aumenta com a circulação de pessoas, ou seja, com o designado "desconfinamento". Sabendo que os contactos próximos

${ }^{1}$ Morada para correspondência: Regina Ferreira Alves, Campus de Gualtar, 4710-057 Braga, Portugal. E-mail: rgnalves@gmail.com 
podem contribuir para aumentar a propagação da infeção, é fundamental quebrar estas cadeias de transmissão (SNS, 2020).

Após um período de emergência nacional, em que as aulas do Ensino Secundário decorreram em regime não-presencial, por meios digitais, exigindo um maior grau de compromisso e disciplina por parte dos/as estudantes, o Ministério da Educação Português decretou o regresso às aulas presenciais dos/as estudantes do $11 .^{\circ}$ e $12 .^{\circ}$ anos no dia 18 de maio de 2020. A tomada desta medida foi pressionada pelo peso que os exames finais nacionais têm na vida académica dos/as estudantes, nomeadamente como prova de ingresso exigida para o acesso ao Ensino Superior (República Portuguesa, 2020). Com o objetivo de implementar medidas de Saúde Pública que evitem a propagação do vírus nesse contexto, a DireçãoGeral da Saúde (DGS) emitiu diversas orientações com medidas de prevenção da transmissão em estabelecimentos de ensino (DGS, 2020d). Resumidamente, entre as principais medidas estão: a garantia de que as escolas apresentam as condições sanitárias necessárias para a promoção das boas práticas de higiene, nomeadamente a higienização das mãos com água e sabão, e secagem com toalhetes de papel; a obrigatoriedade de utilização de máscaras para acesso e permanência nas escolas, pelos/as funcionários/as docentes e não docentes e pelos/as estudantes; a afixação de cartazes disponibilizados pela DGS sobre a correta higienização das mãos, etiqueta respiratória e colocação da máscara; assegurar a limpeza das superfícies de utilização comum várias vezes ao dia.

Vários fatores podem contribuir para explicar os diferentes comportamentos dos/as estudantes em relação à prevenção da COVID-19. A forma como os indivíduos aderem a medidas de promoção da saúde, como estas, é vastamente estudada na área da psicologia da saúde. Um dos fatores mais abordados é o conhecimento ou informação que os indivíduos têm sobre uma determinada doença. De acordo com o modelo de informação, motivação e competências comportamentais (Information, Motivation, Behavioral Skills - IMB) (Fisher \& Fisher, 1992; 1996; 2000;), originalmente desenvolvido no âmbito da compreensão dos determinantes psicológicos da exposição ao risco de HIV e da sua prevenção, se os indivíduos estiverem bem informados, motivados para agir e possuírem as competências comportamentais necessárias, tenderão a iniciar e manter comportamentos saudáveis. Pelo contrário, indivíduos pouco informados, sem motivação para a ação e sem as competências comportamentais requeridas para iniciar a ação efetiva, tenderão a ter comportamentos de risco para a sua saúde. A informação pode consistir em factos específicos que promovam a saúde e que sejam facilmente reconhecidos pelo indivíduo. A importância deste fator é também valorizada pela Teoria da Ação Racional, inicialmente desenvolvida nos anos 60 por Fishbein (1963) e depois aprofundada em colaboração com Ajzen (Fishbein \& Ajzen, 1974, 1975). Esta teoria considera que os indivíduos são seres eminentemente racionais, que utilizam a informação e o conhecimento disponível para desenvolver intenções e comportamentos. Desta forma, ter conhecimento sobre os sintomas de COVID-19, a gravidade da doença, as formas de contágio e as estratégias de prevenção será um promotor da adoção de comportamentos preventivos. Contudo, uma melhor compreensão do comportamento humano exige que se identifique como se formam as intenções comportamentais, que têm por base atitudes pessoais (a avaliação das consequências do comportamento e das crenças comportamentais) e a norma subjetiva em relação a uma determinada ação (as expectativas que outras pessoas significativas possuem em relação ao nosso comportamento e o quanto valorizamos essas expectativas). A importância da inclusão das atitudes e da influência social nos comportamentos é assim realçada por esta teoria, salientando que apenas o aumento do conhecimento, por si só, pode não ser suficiente para provocar a mudança comportamental (Ajzen, 2002).

Com base no modelo IMB, as atitudes são consideradas um fator de "motivação" para iniciar uma determinada ação. Assim, a motivação (ou a sua ausência) pode fazer com que pessoas informadas se envolvam (ou não) em ações de promoção da saúde. Esta inclui uma dimensão pessoal (motivação pessoal), que se refere às atitudes quanto a um determinado comportamento preventivo -, e uma dimensão social (motivação social), relativa ao suporte social percebido em relação a esses comportamentos (Fisher et al., 2003). Assim, ter atitudes favoráveis a medidas preventivas como o uso de máscara e a lavagem cuidadosa das mãos, e percecionar que as Autoridades de Saúde tomam as necessárias ações de proteção dos cidadãos são, por exemplo, fatores promotores da adesão aos comportamentos de prevenção. Outros modelos acrescentam dimensões cognitivas/percetivas como influenciadoras das atitudes e comportamentos. Nesta linha, um dos modelos mais utilizados em promoção da saúde durante as últimas três décadas, e que inclui um importante componente cognitivo é o Modelo de Crenças de Saúde, desenvolvido inicialmente por Rosenstock $(1966 ; 2005)$ e depois revisto por Becker (1974) e Janz e Becker (1984). Este modelo sugere que a probabilidade de um indivíduo adotar um determinado comportamento de saúde é influenciada pelos seguintes elementos fundamentais: suscetibilidade percebida (refere-se à perceção subjetiva do risco de adquirir a doença, neste caso, ter perceção de que, por exemplo, a permanência em espaços públicos deve ser restrita para 
diminuir o risco de contágio por COVID); a gravidade percebida (refere-se ao nível de severidade que uma pessoa atribui a uma doença depois de a contrair, o que muitas vezes tem em conta as consequências médicas da doença bem como as sociais, por exemplo, estar consciente do perigo de contrair o vírus); os benefícios percebidos (perceção da efetividade das várias ações disponíveis para reduzir o risco de contrair a doença, como, por exemplo, considerar que o uso de equipamentos de proteção ajuda a prevenir a infeção); as barreiras percecionadas (os obstáculos à realização de uma determinada ação preventiva que é recomendada); as pistas para a ação (estímulos, internos ou externos, que desencadeiam o processo de tomada de decisão para tomar uma determinada ação); e o sentimento de autoeficácia (nível de confiança para a realização de um determinado comportamento).

As competências comportamentais focam-se, de acordo com o modelo IMB, na capacidade de realizar um determinado comportamento e no seu sentimento de autoeficácia (por exemplo, na prevenção do HIV, pode ser a capacidade de negociar com um/a parceiro/a a utilização de preservativo) (Fisher \& Fisher, 1996; Fisher et al., 2003).

Num contexto de mudança regulatória, com estudantes e professores/as portugueses/as a iniciar o "desconfinamento", é fundamental compreender o seu nível de conhecimento, as suas atitudes e os comportamentos adotados para reduzir o risco relacionado com a sua conduta e que pode ter um acentuado impacto na transmissão da COVID-19. Destes comportamentos são exemplo o uso de máscara, a desinfeção das mãos e o afastamento físico. Procurando incorporar algumas das dimensões da teoria da ação racional, e dos modelos de crenças da saúde e da informação, motivação e competências comportamentais, este estudo teve como objetivos analisar os conhecimentos, as atitudes e os comportamentos relativos à pandemia de COVID-19, numa amostra de estudantes portugueses/as do Ensino Secundário.

\section{MÉTODO}

\section{Participantes}

Realizou-se um estudo transversal e descritivo com estudantes matriculados/as no Ensino Secundário em escolas públicas portuguesas. Entre 7 e 17 de maio de 2020, período em que as escolas se encontravam encerradas devido ao estado de emergência, aplicou-se um questionário previamente validado, anónimo e em formato online a uma amostra não probabilística $(N=1258)$ de estudantes do Ensino Secundário

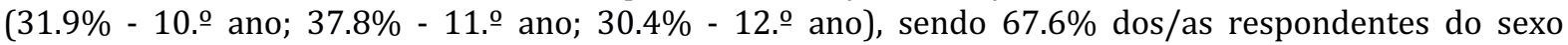
feminino (Tabela 4). A maioria dos/as estudantes tem Portugal como país de origem (95.5\%) e reside na região Norte de Portugal (50.8\%), seguindo-se aqueles/as que residem na região “Lisboa e Tejo" (14.1\%). Mais de metade dos/as estudantes frequenta um curso da área de Ciências e Tecnologias (54.5\%) e $11.1 \%$ frequentam cursos profissionais.

\section{Instrumentos}

O questionário final contém 52 questões; 5 de caráter sociodemográfico (sexo, ano de escolaridade, país de origem, região onde mora e curso) e 47 questões divididas pelas 3 dimensões em estudo:

Conhecimentos sobre a COVID. Esta escala consiste em 14 itens relacionados com o uso de equipamentos de proteção individual, lavagem das mãos, desinfeção, exposição a COVID-19, sintomas e transmissão). Foi pedido aos/às participantes que respondessem se consideravam as afirmações como Verdadeiras, Falsas ou Não sei. Atribuiu-se 1 ponto a cada resposta correta e 0 pontos a uma resposta incorreta ou Não sei. Fez-se o somatório de todos os itens, sendo que pontuações mais altas correspondem a um maior nível de conhecimento. 0 alfa de Cronbach na amostra foi de .519;

Atitudes em relação à COVID-19. Esta escala é composta por 20 itens organizados em 3 fatores (atitudes em relação às medidas preventivas, motivação social e perceção de risco), respondidos através de uma escala de Likert de 5 pontos (de 1 - Discordo Totalmente a 5 - Concordo Totalmente), correspondendo o score mais elevado a atitudes mais positivas face às medidas preventivas, maior motivação social para aderir a comportamentos de saúde e perceção de risco mais elevada. Alguns itens da escala foram invertidos para análise. As características psicométricas da escala foram testadas através da análise da confiabilidade e da análise fatorial em componentes principais, conforme apresentado no Procedimento. De acordo com a análise do Scree Plot e o critério teórico, optou-se pela extração de três fatores, que explicam 37.17\% da variabilidade total $\left(\mathrm{KMO}=.810 ; \chi^{2}(231)=5652.566, p<.001\right)$. 0 alfa de Cronbach de cada fator foi aceitável (variando entre .587 e .692). Os itens ' 0 uso de equipamentos de proteção individual deverá ser uma decisão pessoal' e 'É aceitável ter sintomas de COVID-19 e andar na 
rua' foram excluídos por apresentarem um peso fatorial inferior a 0.3 , apresentando-se apenas os seus resultados descritivos.

O fator Medidas Preventivas agrega itens relacionados com a atitude dos/as estudantes em relação às ações de prevenção recomendadas bem como a "motivação pessoal" para as executar, em concordância com o modelo IMB (Fisher \& Fisher, 1996). Este fator é composto por 5 itens ('O uso de equipamentos de proteção ajuda a prevenir a infeção'; 'O uso de máscara deve ser obrigatório em todos os locais públicos fechados'; 'A lavagem cuidadosa das mãos é importante para prevenir o contágio por COVID-19'; 'É importante lavar/desinfetar superfícies e objetos que possam estar contaminados'; 'Os/As professores/as deviam falar neste tema nas suas aulas'), sendo que quanto mais elevado o seu score, mais favoráveis as atitudes face às medidas preventivas $(\alpha=.587)$.

O fator Motivação Social, assim designado com base no modelo IMB (Fisher \& Fisher, 1996), preconiza que o suporte social, as normas, a informação veiculada publicamente sobre um determinado comportamento podem ser promotoras ou inibidoras da adoção desse comportamento. Este fator inclui 8 itens ('Estou consciente dos perigos deste vírus'; 'Sinto-me bem informado/a sobre a forma de me prevenir da COVID-19'; 'Os meios de comunicação social fornecem informação suficiente sobre a pandemia'; 'Sinto-me seguro/a porque utilizo as medidas de proteção adequadas'; 'Tenho confiança nas Autoridades de Saúde'; 'As recomendações das Autoridades de Saúde sobre os equipamentos de proteção individual são claras'; Faltam programas educativos para a prevenção da COVID-19'; 'Disponho de recursos financeiros para adquirir materiais de proteção individual'), pelo que os valores mais elevados correspondem a uma maior motivação social para a adoção de comportamentos preventivos $(\alpha=.668)$.

0 fator Perceção de Risco engloba itens relacionados com a suscetibilidade percebida à doença bem como as crenças na severidade da mesma, preconizadas no Modelo de Crenças de Saúde (Janz \& Becker, 1984), e é composto por 7 itens ('Sinto-me inseguro/a se tiver que ir para a escola'; 'As aulas práticas deviam recomeçar presencialmente'; 'Tenho receio de contrair a COVID-19'; 'As aulas presenciais deviam terminar este ano letivo'; 'Considero que se criou um alarmismo maior do que o necessário'; 'Tenho receio que algum familiar ou amigo/a contraia COVID-19'; 'A permanência em espaços públicos deveria ser mais restrita'), sendo que quanto mais elevado o score, maior será a perceção de risco dos/as estudantes $(\alpha=.692)$.

Comportamentos preventivos. Esta escala refere-se aos comportamentos preventivos adotados e inclui 13 itens (relativos ao uso de equipamentos de proteção individual, distanciamento físico, lavagem das mãos e desinfeção). Para a análise desta escala, agruparam-se os dois itens que dizem respeito ao uso de máscara (máscara cirúrgica e máscara comunitária), considerando que os/as participantes eram utilizadores de máscara sempre que reportassem o uso de qualquer uma das duas. Cada item foi respondido através de uma escala de Likert de 5 pontos (de 1 - Nunca a 5 - Sempre), tendo-se atribuído 1 ponto a cada comportamento que é praticado sempre. 0 total de comportamentos praticados foi somado, variando entre 0 e 12, sendo que um score elevado nesta dimensão indica bons comportamentos protetores. 0 índice de confiabilidade da escala (alfa de Cronbach) foi de.739.

\section{Procedimento}

Após definição das dimensões principais a inserir no questionário, tendo por base os modelos de promoção da saúde apresentados e sua robustez empírica na predição e promoção de comportamentos preventivos noutras áreas da saúde (Fishbein \& Ajzen, 1974; Fisher et al., 2003; Janz \& Becker, 1984), o instrumento utilizado no presente estudo foi desenvolvido com recurso a uma revisão da literatura, incluindo: a) estudos que avaliassem conhecimentos, atitudes e/ou comportamentos relativos a COVID19 e outras doenças transmissíveis em adolescentes (Chen et al., 2020; Mak \& Lai, 2012) e em estudantes do ensino superior (Alzoubi et al., 2020; Asaad et al., 2019); b) informação sobre a COVID-19, sintomas, transmissão e diretrizes para a sua prevenção emitidas por Autoridades de Saúde (DGS, 2020c; WHO, 2020b). Os itens comummente utilizados para cada uma das dimensões (conhecimentos, atitudes e comportamentos) foram agrupados e, seguidamente, eliminados aqueles que eram redundantes. Duas investigadoras nas áreas das ciências da saúde e ciências da educação reviram uma versão preliminar do instrumento, validando o seu conteúdo. Posteriormente, o questionário foi formatado nos formulários da Google, incluindo um termo de consentimento informado. Realizou-se um estudo piloto com uma amostra de 12 estudantes para testar a sua compreensão e dificuldade. Todos os itens se mantiveram sem alterações.

Um investigador da equipa enviou um convite para colaboração no estudo a todos/as os/as diretores/as das escolas secundárias do país, incluindo as Regiões Autónomas dos Açores e da Madeira, juntamente com o link para aceder ao questionário online, acompanhado do consentimento informado e informação sobre o estudo. Os/as diretores/as das escolas secundárias que aceitaram participar 
divulgaram o link e informação sobre o estudo pelos/as estudantes, de acordo com os regulamentos internos de cada estabelecimento de ensino, garantindo-se a confidencialidade, o anonimato e a participação voluntária.

As análises estatísticas foram realizadas usando o IBM Statistical Package for the Social Sciences (SPSS), versão 26.0, Armonk, NY, USA. Começamos por apresentar os resultados descritivos para cada item das escalas dos conhecimentos, atitudes e comportamentos, em frequências absolutas $(n)$ e relativas (\%). Para analisar as características psicométricas das escalas, analisámos a sua confiabilidade com recurso ao cálculo do alfa de Cronbach $(\alpha)$. Para a escala das atitudes, por conter itens que se referem a áreas distintas, foi realizada uma análise fatorial exploratória, por meio da análise de componentes principais, seguida de rotação ortogonal do tipo varimax (com normalização de Kaiser) para valores próprios iguais ou superiores a 1. Para avaliar as diferenças entre as dimensões de conhecimentos, atitudes e comportamentos em relação à COVID-19 e as características sociodemográficas, utilizámos as medidas contínuas das escalas (média $(M)$ e desvio padrão $(D P)$ ) para realizar o Teste-T para amostras independentes ou a ANOVA, conforme apropriado. Por fim, calculou-se um modelo linear generalizado para determinar as variáveis preditoras dos comportamentos preventivos. 0 nível de significância considerado foi de.05.

\section{RESULTADOS}

\section{Conhecimentos sobre a COVID-19}

Os/as estudantes revelaram bons conhecimentos sobre a COVID-19, visto que grande parte dos itens (10/14) apresenta mais de $90 \%$ de respostas corretas. 0 item "A perda de olfato é um sintoma de COVID19 ” foi aquele onde se registou uma menor proporção de respostas corretas (44.6\%) (Tabela 1).

Tabela 1. Proporção de respostas corretas nos itens relativos aos conhecimentos sobre a COVID-19

\begin{tabular}{lr}
\hline Itens & n (\%) \\
\hline $\begin{array}{l}\text { A etiqueta respiratória (espirrar ou tossir para o antebraço) ajuda a reduzir a exposição e transmissão } \\
\text { de COVID-19. }\end{array}$ & $1138(94.0)$ \\
O uso de máscara facial não ajuda na prevenção da COVID. & $1172(93.2)$ \\
É fundamental manter uma distância de segurança de pelo menos 2 metros para evitar a infeção por & $1202(95.5)$ \\
COVID-19. & 1254 (99.7) \\
As medidas para impedir a propagação da doença incluem a lavagem frequente das mãos. & $1185(94.2)$ \\
A COVID-19 pode transmitir-se através do contacto com superfícies e objetos contaminados. & $1215(96.6)$ \\
As pessoas que correm maior risco de doença grave por COVID-19 são os idosos e pessoas com & $1222(97.1)$ \\
doenças crónicas. & $1032(82.0)$ \\
Os jovens não correm o risco de ser infetados pelo vírus da COVID-19. & $561(44.6)$ \\
A COVID-19 é o mesmo que uma gripe sazonal. & $1192(94.8)$ \\
A perda de olfato é um sintoma de COVID-19. & 1092 (86.8) \\
É possível estar infetado/a com COVID-19 e não ter qualquer tipo de sintoma. & $1236(98.3)$ \\
Todos os casos confirmados de COVID-19 necessitam de internamento. & 1005 (79.9) \\
A tosse, febre ou falta de ar são os principais sintomas de COVID-19. & 1161 (92.3) \\
A COVID-19 apenas se transmite por contacto próximo com pessoas infetadas. & É possível contrair COVID-19 pelo contacto com objetos contaminados. \\
\hline
\end{tabular}

\section{Atitudes em relação à COVID-19}

A maioria dos/as inquiridos/as revelou atitudes positivas quanto às medidas preventivas, como o uso de equipamentos de proteção individual, concordando ou concordando totalmente que a sua utilização ajuda a prevenir a infeção por COVID-19 (94.5\%) e com o uso obrigatório de máscara em todos os espaços fechados (93.2\%). A quase totalidade dos/as estudantes considera importante a lavagem das mãos (95.3\%) e a desinfeção de superfícies (95.46\%) e não acha aceitável que pessoas com sintomas da COVID19 possam andar na rua (94.7\%) (Tabela 2$)$.

A maioria dos/as respondentes revelou confiança nas Autoridade de Saúde (66.9\%) e sentiu-se segura pelo facto de utilizarem medidas de proteção adequadas (72.7\%). No entanto, quando confrontados/as com a questão "Sinto-me inseguro/a se tiver que ir para a escola", a maioria dos/as estudantes (56.0\%) indicou concordar ou concordar totalmente com a afirmação. No mesmo sentido, 66.9\% dos/as estudantes sinalizou ter receio de contrair o vírus, mas um receio ainda maior que algum familiar ou amigo/a o possa contrair (89.0\%), revelando consciência face aos perigos da pandemia (93.7\%). 
A maioria dos/as estudantes concordou ou concordou totalmente com o término das aulas presenciais (55.8\%). Cerca de um terço dos/as estudantes (29.0\%) considera que faltam programas educativos acerca da prevenção da COVID-19, ainda que a maioria (86.6\%) se considere bem informada sobre as formas de prevenção.

Tabela 2. Proporção de concordância (Concordo e Concordo Totalmente) face a cada um dos itens sobre atitudes em relação à COVID-19

\begin{tabular}{|c|c|}
\hline Itens & $n(\%)$ \\
\hline O uso de equipamentos de proteção individual deverá ser uma decisão pessoal. & $131(10.4)$ \\
\hline O uso de equipamentos de proteção ajuda a prevenir a infeção. & 1189 (94.5) \\
\hline O uso de máscara deve ser obrigatório em todos os locais públicos fechados. & $1173(93.2)$ \\
\hline A lavagem cuidadosa das mãos é importante para prevenir o contágio por COVID-19. & 1199 (95.3) \\
\hline É importante lavar/desinfetar superfícies e objetos que possam estar contaminados. & $1203(95.6)$ \\
\hline $\begin{array}{l}\text { As recomendações das Autoridades de Saúde sobre os equipamentos de proteção individual são } \\
\text { claras. }\end{array}$ & $856(68.0)$ \\
\hline A permanência em espaços públicos deveria ser mais restrita. & $795(63.2)$ \\
\hline É aceitável ter sintomas de COVID-19 e andar na rua. & $26(2.1)$ \\
\hline Tenho confiança nas Autoridades de Saúde. & $842(66.9)$ \\
\hline Sinto-me seguro/a porque utilizo as medidas de proteção adequadas. & $914(72.7)$ \\
\hline Sinto-me inseguro/a se tiver que ir para a escola. & $705(56.0)$ \\
\hline Tenho receio de contrair a COVID-19. & $842(66.9)$ \\
\hline Tenho receio que algum familiar ou amigo/a contraia COVID-19. & $1120(89.0)$ \\
\hline Estou consciente dos perigos deste vírus. & 1179 (93.7) \\
\hline As aulas presenciais deviam terminar este ano letivo. & $702(55.8)$ \\
\hline As aulas práticas deviam recomeçar presencialmente. & $226(18.0)$ \\
\hline Considero que se criou um alarmismo maior do que o necessário. & $186(14.8)$ \\
\hline Os meios de comunicação social fornecem informação suficiente sobre a pandemia. & $721(57.3)$ \\
\hline Disponho de recursos financeiros para adquirir materiais de proteção individual. & $905(71.9)$ \\
\hline Faltam programas educativos para a prevenção da COVID-19. & $365(29.0)$ \\
\hline Os/As professores/as deviam falar neste tema nas suas aulas. & $601(47.8)$ \\
\hline Sinto-me bem informado/a sobre a forma de me prevenir da COVID-19. & $1093(86.9)$ \\
\hline
\end{tabular}

\section{Comportamentos relativos à COVID-19}

Relativamente ao uso de equipamentos de proteção individual, 80.3\% referiu utilizar sempre máscara cirúrgica ou comunitária em espaços fechados. As luvas descartáveis nunca são usadas por cerca de um quarto dos/as inquiridos/as (28.9\%) e 79.5\% nunca usa viseira em locais fechados (Tabela 3).

A maioria dos/as estudantes selecionou a opção "Sempre" quando questionada se evitava interações sociais que implicavam proximidade $(62.3 \%)$ e se mantinha a distância de segurança durante caminhadas ou prática de desporto ao ar livre (68.0\%). No entanto, apenas 34.2\% referiu cumprir esta distância quando conversa com alguém. A lavagem das mãos, de acordo com as diretrizes promovidas pelas Autoridades de Saúde, é reportada por $62.2 \%$ dos/as estudantes. 
Tabela 3. Proporção de comportamentos preventivos realizados "Sempre" pelos/as estudantes

\begin{tabular}{lr}
\hline Itens & $\boldsymbol{n}(\%)$ \\
\hline Com que frequência... & $1010(80.3)$ \\
Usa máscara (cirúrgica ou comunitária) de proteção em locais fechados? & $439(34.9)$ \\
Coloca máscara logo que sai de casa? & $411(32.7)$ \\
Usa luvas descartáveis em locais fechados? & $50(4.0)$ \\
Usa viseira em locais fechados? & $430(34.2)$ \\
Quando está a falar com alguém, mantém a distância de segurança (mínimo de 2 metros)? & $856(68.0)$ \\
Quando caminha ou faz desporto ao ar livre, mantém a distância de segurança? & $784(62.3)$ \\
Evita interações sociais que impliquem proximidade física? & $783(62.2)$ \\
Lava as mãos de acordo com as diretrizes da DGS (com água e sabão cerca de 20 segundos)? & $687(54.6)$ \\
Usa álcool ou loções desinfetantes (como álcool-gel, por exemplo)? & $719(57.2)$ \\
Desinfeta e/ou muda o seu calçado quando chega a casa? & $457(36.3)$ \\
Desinfeta os produtos que compra? & $340(27.0)$ \\
Desinfeta objetos como maçanetas, puxadores, torneiras, ou corrimões, em sua casa?
\end{tabular}

\section{Conhecimentos, atitudes e comportamentos em relação à COVID-19 em função das características sociodemográficas dos/as estudantes}

Os/As estudantes revelaram bons conhecimentos sobre a COVID-19, respondendo corretamente, em média, a $12.49 \pm 1.45$ itens (num total de 14). Verificou-se a inexistência de diferenças entre o nível de conhecimento e o sexo dos/as estudantes, $t(1256)=-.946, p>.05$. Os/as estudantes que frequentam o 12. . ano revelaram um maior nível de conhecimento $(M=12.66, D P=1.25)$ em comparação com aqueles/as que frequentam o $10 . \mathrm{o}$ ano $(M=12.49, D P=1.51), Z(2,1255)=3.741, p<.05$. Adicionalmente, os/as estudantes que residem na região Centro apresentaram um maior nível de conhecimento $(M=$ $12.95, D P=1.50)$ em comparação com os/as da região do Algarve $(M=12.05, D P=1.62), Z(6,1244)=$ $4.730, p<.001$. Para além disso, os/as estudantes que frequentam um curso da área das Ciências e Tecnologias $(M=12.74, D P=1.33)$ revelaram um nível de conhecimento superior aos/às estudantes que frequentam cursos profissionais $(M=11.82, D P=1.78), Z(4,1231)=15.622, p<.001$, (Tabela 4$)$. 
Tabela 4. Conhecimentos, atitudes e comportamentos preventivos em função do sexo, ano de escolaridade, região e curso dos/as estudantes

\begin{tabular}{|c|c|c|c|c|c|c|c|c|c|c|c|c|}
\hline & & \multirow{3}{*}{$n(\%)$} & \multirow{2}{*}{\multicolumn{2}{|c|}{$\begin{array}{c}\text { Conhecimentos } \\
\quad(0-14)\end{array}$}} & \multicolumn{6}{|c|}{$\begin{array}{l}\text { Atitudes em relação à COVID-19 } \\
(0-5)\end{array}$} & \multirow{2}{*}{\multicolumn{2}{|c|}{$\begin{array}{c}\text { Comportamentos } \\
\text { Preventivos } \\
(0-12) \\
\end{array}$}} \\
\hline & & & & & \multicolumn{2}{|c|}{ Medidas Preventivas } & \multicolumn{2}{|c|}{ Motivação Social } & \multicolumn{2}{|c|}{ Perceção de Risco } & & \\
\hline & & & $M(D P)$ & $t / F$ & $M(D P)$ & $t / F$ & $M(D P)$ & $t / F$ & $M(D P)$ & $t / F$ & $M(D P)$ & $t / F$ \\
\hline \multirow[t]{2}{*}{ Sexo } & Masculino & $408(32.43)$ & $12.43(1.58)$ & \multirow{2}{*}{-.946} & $4.48(.53)$ & \multirow{2}{*}{-1.192} & $3.91(.58)$ & \multirow{2}{*}{-.836} & $3.76(.76)$ & \multirow{2}{*}{$-4.220 * * *$} & $5.45(2.98)$ & \multirow{2}{*}{$-4.063 * * *$} \\
\hline & Feminino & $850(67.57)$ & $12.52(1.38)$ & & $4.52(.47)$ & & $3.94(.55)$ & & $3.94(.69)$ & & $6.15(2.78)$ & \\
\hline \multirow{3}{*}{$\begin{array}{l}\text { Ano de } \\
\text { escolaridade }\end{array}$} & 10. ano & $401(31.88)$ & $12.40(1.51)$ & & $4.52(.44)$ & & $4.00(.51)$ & & $3.92(.70)$ & & $5.88(2.84)$ & \\
\hline & 11.o ano & $475(37.76)$ & $12.43(1.54)$ & $3.741 *$ & $4.50(.51)$ & .385 & $3.90(.58)$ & 5.800 ** & $3.88(.74)$ & .643 & $5.90(2.81)$ & .197 \\
\hline & 12. ano & $382(30.37)$ & $12.66(1.25)$ & & $4.40(.49)$ & & $3.88(.56)$ & & $3.86(.71)$ & & $6.00(2.95)$ & \\
\hline \multirow[t]{7}{*}{ Região } & Norte & $635(50.76)$ & $12.64(1.37)$ & & $4.57(.43)$ & & $3.91(.55)$ & & $3.94(.69)$ & & $6.18(2.77)$ & \\
\hline & Centro & $41(3.28)$ & $12.95(1.50)$ & & $4.46(.43)$ & & $3.79(.62)$ & & $3.85(.61)$ & & $5.71(2.90)$ & \\
\hline & Área Metropolitana de Lisboa & $176(14.07)$ & $12.38(1.52)$ & & $4.46(.45)$ & & $3.93(.53)$ & & $3.85(.65)$ & & $5.74(2.88)$ & \\
\hline & Alentejo & $23(1.84)$ & $12.35(1.72)$ & $4.730 * * *$ & $4.43(.43)$ & $3.336^{* *}$ & $4.04(.49)$ & 1.533 & $3.83(.77)$ & 1.423 & $5.65(2.12)$ & $2.350 *$ \\
\hline & Algarve & $136(10.87)$ & $12.05(1.62)$ & & $4.43(.56)$ & & $3.88(.58)$ & & $3.83(.83)$ & & $5.67(2.87)$ & \\
\hline & Região Autónoma dos Açores & 135(10.79) & $12.28(1.42)$ & & $4.43(.61)$ & & $3.98(.54)$ & & $3.84(.79)$ & & $5.30(3.16)$ & \\
\hline & Região Autónoma da Madeira & 105(8.39) & $12.54(1.34)$ & & $4.46(.54)$ & & $4.02(.58)$ & & $3.77(.75)$ & & $6.00(2.96)$ & \\
\hline \multirow[t]{5}{*}{ Curso } & Ciências e Tecnologias & $674(54.5)$ & $12.74(1.33)$ & & $4.52(.43)$ & & $3.90(.48)$ & & $3.88(.70)$ & & $5.71(2.75)$ & \\
\hline & Artes Visuais & $62(5.0)$ & $12.19(1.44)$ & & $4.51(.44)$ & & $3.93(.55)$ & & $3.87(.67)$ & & $6.77(2.87)$ & \\
\hline & Ciências Socioeconómicas & $129(18.9)$ & $12.49(1.23)$ & $15.622^{* * *}$ & $4.54(.44)$ & $3.824^{* *}$ & $3.85(.49)$ & .764 & $3.95(.63)$ & .644 & $5.95(2.91)$ & $5.121 * * *$ \\
\hline & Línguas e Humanidades & $234(19.8)$ & $12.24(1.44)$ & & $4.53(.52)$ & & $3.93(.54)$ & & $3.89(.78)$ & & $6.48(2.89)$ & \\
\hline & Profissional & $137(11.1)$ & $11.82(1.78)$ & & $4.35(.70)$ & & $3.85(.60)$ & & $3.81(.79)$ & & $5.58(3.14)$ & \\
\hline Total & & $1258(100.0)$ & $12.49(1.45)$ & & $4.51(.49)$ & & $3.93(.56)$ & & $3.89(.72)$ & & $5.92(2.86)$ & \\
\hline
\end{tabular}

$M$, média; $D P$, desvio padrão; $F$, ANOVA

${ }^{* * *} p<.001$; $^{* *} p<.01 ; * p<.05$ 
Relativamente à escala das atitudes em relação à COVID-19, os/as estudantes mostraram atitudes altamente favoráveis face às medidas preventivas $(M=4.51, D P=.49)$, sendo os/as estudantes da Região Norte aqueles/as que apresentaram atitudes mais favoráveis em comparação com os/as estudantes das outras regiões do país, $Z(6,1244)=3.336, p<.01$. Por sua vez, os/as estudantes dos cursos profissionais apresentaram atitudes mais negativas face às medidas preventivas $(M=4.35, D P=.70)$ do que os/as estudantes que frequentam cursos científico-humanísticos, $Z(4,1231)=3.824, p<.01$. Na dimensão de motivação social, os/as estudantes inquiridos/as consideraram que a informação veiculada publicamente sobre os riscos da COVID-19 bem como as recomendações da Autoridades de Saúde promovem a adoção de comportamentos preventivos $(M=3.93, D P=.56)$, sendo os/as estudantes a frequentar o $10 .$. ano de escolaridade aqueles/as que revelaram maior motivação social para aderir a esses comportamentos, $Z(2,1255)=5.800, p<.01$.

A perceção de risco entre os/as estudantes pode considerar-se elevada $(M=3.89, D P=.72)$, encontrando-se diferenças no nível de perceção entre estudantes do sexo masculino e do sexo feminino, $t(1256)=-4.220, p<.01)$ Assim, as estudantes $(M=3.94, D P=.69)$ evidenciaram uma perceção de risco ligeiramente superior à dos estudantes $(M=3.76, D P=.76)$ (Tabela 4).

No que se refere aos comportamentos preventivos, considerando os doze comportamentos apresentados, verificou-se que, em média, os/as estudantes relataram adotar sempre $5.92 \pm 2.86$ desses comportamentos. 0 score dos comportamentos é superior nas inquiridas $(M=6.15, D P=2.78) \mathrm{em}$ comparação com os estudantes do sexo masculino $(M=5.45, D P=2.98)$, sendo essas diferenças estatisticamente significativas, $t(1256)=-4.063, p<.001$. Adicionalmente, os/as estudantes que residem na região Norte $(M=6.18, D P=2.77)$ apresentaram uma média de comportamentos preventivos superior à dos/as estudantes residentes na Região Autónoma dos Açores $(M=5.30, D P=3.16), Z(2,1255)=2.350$, $p<.05$. Por último, os/as estudantes do Curso de Artes Visuais $(M=6.77, D P=2.87)$ apresentaram uma média superior de comportamentos preventivos em comparação com os/as estudantes de Ciências e Tecnologias $(M=5.71, D P=2.75)$ e os/as dos Cursos Profissionais $(M=5.58, D P=3.14), Z(4,1231)=$ $5.121, p<.001$.

De acordo com os resultados do modelo linear generalizado, o sexo dos estudantes, o curso e as dimensões da escala de atitudes em relação à COVID-19 tiveram um efeito estatisticamente significativo nos comportamentos preventivos dos/as estudantes. Assim, ser do sexo feminino, $\operatorname{Exp}(\beta)=1.714, p<$ .001 , e frequentar cursos de Artes Visuais, $\operatorname{Exp}(\beta)=2.866, p<.01$, ou de Línguas e Humanidades, $\operatorname{Exp}(\beta)=$ 2.506, $p<.001$, aumenta as probabilidades de adotar comportamentos preventivos. Paralelamente, atitudes mais positivas em relação à prevenção da COVID-19 predizem um maior número de comportamentos adotados, $\operatorname{Exp}(\beta)_{\text {Medidas Preventivas }}=1.223, p<.001 ; \operatorname{Exp}(\beta)_{\text {Motivação Social }}=1.164, p<.001$; $\operatorname{Exp}(\beta)$ Perceção de risco $=1.131, p<.001$ (Tabela 5). 
Tabela 5. Modelo Linear Generalizado sobre os fatores preditores dos Comportamentos Preventivos

\begin{tabular}{|c|c|c|c|c|c|c|c|c|}
\hline & B & SE & $\chi^{2}$ Wald & df & $p$ & $\operatorname{Exp}(B)$ & \multicolumn{2}{|c|}{$95 \%$ CI } \\
\hline Constante & -4.346 & .9833 & 19.533 & 1 & .000 & .013 & .002 & .089 \\
\hline \multicolumn{9}{|l|}{ Sexo } \\
\hline Masculino & 1 & . & . & . & . & 1 & . & . \\
\hline Feminino & .539 & .1634 & 10.870 & 1 & .001 & 1.714 & 1.244 & 2.361 \\
\hline \multicolumn{9}{|l|}{ Ano de escolaridade } \\
\hline $10 . \stackrel{\circ}{\text { ano }}$ & 1 & . & . & . & . & 1 & . & . \\
\hline $11 .^{\circ}$ ano & .090 & .1885 & .230 & 1 & .631 & 1.095 & .757 & 1.584 \\
\hline 12. $\stackrel{\circ}{\text { ano }}$ & .318 & .1957 & 2.640 & 1 & .104 & 1.374 & .936 & 2.017 \\
\hline \multicolumn{9}{|l|}{ Regiões } \\
\hline Norte & 1 & . & . & . & . & 1 & . & . \\
\hline Centro & .036 & .4599 & .006 & 1 & .937 & 1.037 & .421 & 2.554 \\
\hline Área Metropolitana de Lisboa & -.443 & .2294 & 3.723 & 1 & .054 & .642 & .410 & 1.007 \\
\hline Alentejo & -.264 & .5718 & .213 & 1 & .644 & .768 & .250 & 2.356 \\
\hline Algarve & -.534 & .2572 & 4.303 & 1 & .038 & .586 & .354 & .971 \\
\hline Região Autónoma dos Açores & -.989 & .2647 & 13.954 & 1 & .000 & .372 & .221 & .625 \\
\hline Região Autónoma da Madeira & -.166 & .2800 & .352 & 1 & .553 & .847 & .489 & 1.466 \\
\hline \multicolumn{9}{|l|}{ Curso } \\
\hline Ciências e Tecnologias & 1 & . & . & . & . & 1 & . & . \\
\hline Artes Visuais & 1.053 & .3538 & 8.858 & 1 & .003 & 2.866 & 1.433 & 5.734 \\
\hline Ciências Socioeconómicas & .196 & .2554 & .587 & 1 & .444 & 1.216 & .737 & 2.006 \\
\hline Línguas e Humanidades & .919 & .2094 & 19.250 & 1 & .000 & 2.506 & 1.662 & 3.777 \\
\hline Profissional & .137 & .2602 & .276 & 1 & .599 & 1.147 & .688 & 1.909 \\
\hline Conhecimento & .001 & .0555 & .000 & 1 & .990 & 1.001 & .898 & 1.116 \\
\hline \multicolumn{9}{|l|}{ Atitudes em relação à COVID-19 } \\
\hline Medidas preventivas & .695 & .1833 & 14.390 & 1 & .000 & 2.004 & 1.399 & 2.871 \\
\hline Motivação social & 1.026 & .1639 & 39.204 & 1 & .000 & 2.791 & 2.024 & 3.849 \\
\hline Perceção de risco & .673 & .1120 & 36.144 & 1 & .000 & 1.961 & 1.574 & 2.443 \\
\hline
\end{tabular}

AIC $=5896.685 ; \chi^{2}(17)=216.248, p<.001$

\section{DISCUSSÃO}

Este estudo é um dos primeiros a avaliar conhecimentos, atitudes e comportamentos em relação à COVID19 numa amostra de estudantes do Ensino Secundário em Portugal. Globalmente, os resultados obtidos revelaram que os/as estudantes têm bons conhecimentos no que respeita à natureza da doença, às vias de transmissão do vírus e às suas formas de prevenção. As atitudes dos estudantes são, de uma forma geral, positivas em relação às medidas de prevenção. Contudo, os/as estudantes aderem apenas a cerca de metade dos comportamentos preventivos avaliados no âmbito da infeção por COVID-19. Algumas variáveis parecem estar associadas a uma maior adoção de comportamentos preventivos: ser mulher, frequentar um curso de Artes Visuais ou de Línguas e Humanidades e ter atitudes positivas em relação às medidas preventivas de COVID-19, uma elevada motivação social e elevada perceção do risco. Estes dados podem ser bons indicadores quanto ao cumprimento das recomendações públicas para a prevenção da COVID-19 (Buzzi et al., 2020) e dar pistas para intervenções de promoção da saúde destinadas a esta população. 0 elevado nível de conhecimento sobre a COVID-19 nesta amostra foi também encontrado noutros estudos em amostras de estudantes universitários/as (Gallè et al., 2020) e na população em geral (Abdelhafiz et al., 2020). Ainda que não tenhamos avaliado as fontes de informação, as Autoridades de Saúde e os meios de comunicação social, nomeadamente plataformas online como as redes sociais mais acedidas pelos/as adolescentes, desempenharam neste período um papel importante de divulgação permanente e atualizada sobre o vírus, a gravidade da doença e sintomas, epidemiologia da doença, as formas de transmissão e a prevenção, entre outros tópicos. Os media são assim uma fonte fundamental de aquisição de informação sobre temas de saúde pública (Głabska et al., 2020; Rice \& Atkin, 2013). Como também já reportado em estudos na área da COVID-19 (Gallè et al., 2020) e noutros tópicos de saúde, os/as estudantes de áreas ligadas às ciências naturais apresentam níveis de conhecimento mais elevados 
do que estudantes de outras áreas científicas, salientando-se nos nossos dados os/as estudantes de cursos profissionais. Assim, o investimento na disseminação de informação e promoção de atitudes e comportamentos adequados, em meio escolar, é fundamental e deve ter em conta as especificidades dos públicos-alvo (Moura et al., 2019; Rice \& Atkin, 2013). É importante que a informação proporcionada seja: 1) objetiva e exata, baseada em factos científicos, devidamente documentada e isenta de mensagens moralizadoras; 2) facilmente inteligível por todas as pessoas a quem esta é destinada; 3) coerente; 4) e, por último, a informação com intencionalidade preventiva deve ter um moderado conteúdo emocional (Rice \& Atkin, 2013), sendo desejável que as atitudes formadas se baseiem em emoções positivas geradas por sentimentos de aceitação de valores.

Ao nível das atitudes, os/as estudantes mostraram globalmente atitudes muito positivas em relação às medidas de prevenção recomendadas, como encontrado noutros estudos (Abdelhafiz et al., 2020), assim como quanto à motivação social para o seu cumprimento, revelando confiança na informação que lhes é fornecida pelas Autoridades de Saúde e uma perceção de risco elevada. É assim importante que a Escola continue esta comunicação clara, envolvendo-se numa educação preventiva que mantenha estas atitudes positivas dos/as estudantes. Adicionalmente, são as estudantes do sexo feminino que apresentam uma maior perceção do risco, o que é concordante com estudos prévios (Zhong et al., 2020) e com a socialização de género, que envolve uma certa ideologia associada a que as mulheres sejam mais responsáveis e não se envolvam em comportamentos de risco, enquanto que a masculinidade se associa ao evitamento de fraqueza e vulnerabilidade, espírito de aventura e autossuficiência, o que explica a elevada presença dos homens em indicadores de saúde ligados a práticas de risco (De Visser et al., 2009).

Em relação aos comportamentos, os resultados mostraram uma elevada proporção de estudantes com comportamentos inadequados no âmbito da infeção por COVID-19, o que foi também encontrado noutros estudos, onde se verificou que apesar de os/as participantes considerarem que a máscara protege da infeção, apenas 35\% a utiliza (Abdelhafiz et al., 2020); ou onde adesão à prática de lavagem das mãos foi reportada por apenas 60\% dos/as inquiridos/as (Chen et al., 2020) ou, ainda, onde 12\% dos/as adolescentes referiu fazê-lo raramente (Głabska et al., 2020). Este resultado mostra que, conforme preconizado por Ajzen (2002), o aumento do conhecimento, por si só, pode não ser suficiente para provocar a mudança comportamental. Contudo, a adesão a comportamentos preventivos foi maior nas estudantes do sexo feminino do que no sexo masculino, em consonância com estudos anteriores (Chen et al., 2020), que mostram que as mulheres tendem a correr menos riscos e a tomar ações mais adequadas e cuidadosas no que concerne a proteção da sua saúde (Zhong et al., 2020).

0 facto de os/as estudantes da Região Centro apresentarem níveis mais elevados de conhecimento, e os/as da Região Norte atitudes mais positivas quanto às medidas preventivas, além de adotarem mais comportamentos preventivos (aqui quando comparados com a Região Autónoma dos Açores), pode refletir as características epidemiológicas da pandemia, com incidências diferenciadas ao longo do tempo nas várias regiões do país (DGS, 2020c). A Região Norte foi aquela onde se registaram os primeiros casos e onde se registou o maior número de casos de infeção nas primeiras semanas, o que pode justificar diferentes níveis de alerta na população, maior recetividade à informação e eventualmente ter conhecimento de familiares ou conhecidos/as infetados/as, o que está associado a maior conhecimento sobre COVID-19 e a maior adesão a medidas de controlo da doença (Gallè et al., 2020).

Um dos resultados mais relevantes encontrados neste estudo refere-se à importância das atitudes em relação à COVID-19 na predição dos comportamentos preventivos, ou seja, à medida que as atitudes são mais favoráveis às medidas preventivas, à motivação social para realizar uma ação e a perceção de risco é mais elevada, os comportamentos preventivos aumentam. Tal como no estudo realizado por Oosterhoff e Palmer (2020), as atitudes dos/as adolescentes em relação à gravidade da COVID-19 foram associadas a um maior distanciamento social, desinfeção e monitorização de notícias. De acordo com o Modelo de Crenças na Saúde, os indivíduos têm maior probabilidade de iniciar uma ação se o risco percebido da doença é elevado (Janz \& Becker, 1984), o que acontece neste estudo. Da mesma forma, o facto de terem elevada motivação, tanto pessoal como social, favorável às medidas preventivas da doença (Fisher et al., 2003) contribui para querer realizar essa ação. Também em linha com Fishbein e Ajzen (1974, 1975), a importância das atitudes positivas como preditoras dos comportamentos vem chamar a atenção para a relevância de incluir estas dimensões em campanhas de consciencialização para os perigos da COVID-19. Assim, parece ser de realçar o investimento na criação de ambientes promotores de saúde, quer pela provisão de informação fidedigna pelas instituições, como pela tomada de medidas de saúde pública que contribuam para promover nos cidadãos atitudes de confiança em relação à segurança na utilização de espaços comuns, como as escolas (DGS, 2020d).

Ainda que sejam os/as estudantes de Ciências e Tecnologias aqueles/as que revelam mais conhecimentos, os/as estudantes em cursos das áreas de Artes Visuais e de Línguas e Humanidades têm 
maior probabilidade de enveredar em comportamentos preventivos. Tendo em conta que estes cursos são mais frequentados por estudantes do sexo feminino, este pode ser um fator que esteja a mediar esta relação. Contudo, para melhor compreender estes resultados é necessário que estes sejam futuramente complementados com estudos qualitativos que permitam captar e analisar em profundidade a forma como os indivíduos atribuem significado a esta dimensão da saúde (Teti et al., 2020). Além disso, a inclusão de outras variáveis sociodemográficas e de contexto, como o nível socioeconómico ou o conhecimento de pessoas próximas que tiveram a doença, pode contribuir para uma melhor compreensão das dimensões que influenciam a adesão a comportamentos preventivos da COVID-19.

Este estudo apresenta algumas limitações. Em primeiro lugar, o recurso a uma plataforma online para recolha de dados pode causar algum enviesamento dos dados, nomeadamente na escala dos conhecimentos, visto que nada nos garante que os/as estudantes não tenham procurado as respostas. Além disso, os comportamentos preventivos foram autorrelatados, pelo que podem não corresponder exatamente à forma como os/as estudantes se comportam. Contudo, a recolha de dados online pode ser vantajosa a este nível, por manter a total confidencialidade e anonimato e, assim, reduzir a desejabilidade social. Em segundo lugar, o convite para a participação neste estudo foi realizado de forma indireta, não se garantindo que todos/as os/as estudantes do Ensino Secundário tenham sido convidados/as. Assim, ainda que o tamanho amostral seja elevado, tendo em conta o número de estudantes matriculados/as em Portugal, no ano letivo de 2019/2020, do 10. ao 12. ano (FFMS \& PORDATA, 2020), a amostra deste estudo representa $0.399 \%$ do total de estudantes, o que não permite a generalização dos resultados a todos/as os/as estudantes que frequentam o Ensino Secundário português.

\section{CONCLUSÃO}

Este foi um dos primeiros estudos a descrever, a partir de uma amostra de 1258 estudantes do 10..$^{\circ}$ ao 12. ${ }^{\circ}$ ano de escolaridade, os conhecimentos, as atitudes os e comportamentos de prevenção da COVID-19 em estudantes portugueses/as, com o propósito de contribuir para a estratégia de redução de riscos associados a esta pandemia. Embora a amostra não seja representativa da população do Ensino Secundário em Portugal, os dados obtidos permitiram perceber que o nível de conhecimento dos/as estudantes sobre COVID-19 é elevado; as atitudes em relação aos comportamentos preventivos são maioritariamente favoráveis e estas predizem a adoção dos comportamentos. Há ainda margem para investir na promoção da adesão a comportamentos preventivos, considerando que existem lacunas a este nível.

Com o país a retomar as atividades, é fundamental que se mantenham os comportamentos para reduzir o risco de transmissão da doença (como o uso de máscara, a desinfeção das mãos e o distanciamento físico), não apenas nas escolas como em todos os contextos de interação social. Assim, os esforços na transmissão de informação credível e criação de ambientes que motivem a adoção de medidas preventivas devem manter-se, visto que essa poderá influenciar as atitudes e aumentar os comportamentos de saúde dos/as jovens.

\section{REFERÊNCIAS}

Abdelhafiz, A. S., Mohammed, Z., Ibrahim, M. E., Ziady, H. H., Alorabi, M., Ayyad, M., \& Sultan, E. A. (2020). Knowledge, perceptions, and attitude of Egyptians towards the novel coronavirus disease (COVID19). Journal of Community Health, 45(5), 881-890. https://doi.org/10.1007/s10900-020-00827-7

Ajzen, I. (2002). Perceived behavioral control, selfefficacy, locus of control, and the theory of planned behavior. Journal of Applied Social Psychology, 32, 1-20. https://doi.org/10.1111/j.15591816.2002.tb00236.x

Alzoubi, H., Alnawaiseh, N., Al-Mnayyis, A., Abu- Lubad, M., Aqel, A., \& Al-Shagahin, H. (2020). COVID-19 Knowledge, attitude and practice among medical and non-medical university students in Jordan. Journal of Pure and Applied Microbiology, 14(1), 17-24. https://doi.org/10.22207/JPAM.14.1.04

Asaad, A. M., El-Sokkary, R. H., Aedh, A. I., Ali Alzamanan, M. A., \& Khalil, F. O. (2019). Exploring knowledge and attitude toward Middle East Respiratory Syndrome-Coronavirus (MERS-CoV) among university health colleges' students, Saudi Arabia: A cross-sectional study. American Journal of Infectious Diseases, 15(1), 37-43. https://doi.org/10.3844/ajidsp.2019.37.43

Becker, M. H. (1974). The health belief model and sick role behavior. Health Education Monographs, 2(4), 409-419. https://doi.org/10.1177/109019817400200407

Buzzi, C., Tucci, M., Ciprandi, R., Brambilla, I., Caimmi, S., Ciprandi, G., \& Marseglia, G. L. (2020). The psycho-social effects of COVID-19 on Italian adolescents' attitudes and behaviors. Italian Journal of Pediatrics, 46(1), 4-11. https://doi.org/10.1186/s13052-020-00833-4

Chen, X., Ran, L., Liu, Q., Hu, Q., Du, X., \& Tan, X. (2020). Hand hygiene, mask-wearing behaviors and its associated factors during the COVID-19 epidemic: A cross-sectional study among primary school 
students in Wuhan, China. International Journal of Environmental Research and Public Health, 17(8). https://doi.org/10.3390/ijerph17082893

De Visser, R. O., Smith, J. A., \& McDonnell, E. J. (2009). 'That's not masculine.' Journal of Health Psychology, 14(7), 1047-1058. https://doi.org/10.1177/1359105309342299

DGS - Direção-Geral da Saúde. (2020a). SARS-CoV-2 / COVID-19. Relatório de situação - 1. Retrieved from https://covid19.min-saude.pt/wp-content/uploads/2020/03/Relatório-de-Situação-1.pdf

DGS - Direção-Geral da Saúde. (2020b). Novo coronavírus. COVID-19. Relatório de situação: 15 de setembro de 2020. Retrieved from https://covid19.min-saude.pt/wpcontent/uploads/2020/09/197_DGS_boletim_20200915.pdf

DGS - Direção-Geral da Saúde. (2020c). COVID-19. Retrieved June 3, 2020, from https://covid19.minsaude.pt/

DGS - Direção-Geral da Saúde. (2020d). Orientação no 024/2020 de 08/05/2020. Regresso ao regime presencial dos $11 .^{\circ}$ e $12 .^{\circ}$ anos de escolaridade e dos $2 .^{\circ}$ e $3 .^{\circ}$ anos dos cursos de dupla certificação do ensino secundário. Retrieved from https://covid19.min-saude.pt/orientacoes/

FFMS, \& PORDATA. (2020). Alunos matriculados no ensino público: total e por nível de ensino. Retrieved August 12, 2020, from https://www.pordata.pt/Portugal/Alunos+matriculados+no+ensino+público+total+e+por+nível+d e+ensino-1003-7968

Fishbein, M. (1963). An investigation of the relationships between beliefs about an object and the attitude toward that object. Human Relations, 16, 233-240. https://doi.org/10.1177/001872676301600302

Fishbein, M., \& Ajzen, I. (1974). Attitudes toward objects as predictors of single and multiple behavioral criteria. Psychological Review, 81, 59-74.

Fishbein, M., \& Ajzen, I. (1975). Belief, attitude, intention and behavior: An introduction to theory and research. Massachusetts: Addison-W. Fisher, J. D., \& Fisher, W. A. (1992). Changing AIDS risk behavior. Psychological Bulletin, 111, 455-74. https://doi.org/10.1037/0033-2909.111.3.455

Fisher, J. D., \& Fisher, W. A. (2000). Theoretical approaches to individual level change in HIV risk behavior. In J. L. Peterson \& R. J. DiClemente (Eds.), Handbook of HIV Prevention (pp. 3-55). Kluwer Aca.

Fisher, J. D., \& Fisher, W. A. (1996). The Information-Motivation-Behavioral skills model of AIDS risk behavior change: Empirical support and application. In S. Oskamp \& S. C. Thompson (Eds.), The Claremont Symposium on Applied Social Psychology. Understanding and preventing HIV risk behavior: Safer sex and drug use (pp. 100-127). Sage Publications, Inc.

Fisher, W. A., Fisher, J. D., \& Harman, J. (2003). The information-motivation- behavioral skills model: A General Social Psychological approach to understanding and promoting health behavior. In J. Suls \& K. A. Wallston (Eds.), Social Psychological Foundations of Health and Illness. (pp. 82-106). Blackwell.

Gallè, F., Sabella, E. A., Da Molin, G., De Giglio, O., Caggiano, G., Di Onofrio, V., ... Napoli, C. (2020). Understanding knowledge and behaviors related to CoViD-19 epidemic in Italian undergraduate students: The EPICO study. International Journal of Environmental Research and Public Health, 17(10), 3481. https://doi.org/10.3390/ijerph17103481

Głabska, D., Skolmowska, D., \& Guzek, D. (2020). Population-based study of the influence of the COVID-19 pandemic on hand hygiene behaviors-polish adolescents' COVID-19 experience (place-19) study. Sustainability, 12(12), 4930. https://doi.org/10.3390/SU12124930

Janz, N. K., \& Becker, M. H. (1984). The health belief model: A decade later. Health Education Quarterly, 11(1), 1-47. https://doi.org/10.1177/109019818401100101.Mak, K. K., \& Lai, C. M. (2012). Knowledge, risk perceptions, and preventive precautions among Hong Kong students during the 2009 influenza A (H1N1) pandemic. American Journal of Infection Control, 40(3), 273-275. https://doi.org/10.1016/j.ajic.2011.10.023

Moura, A., Silva, S., de Freitas, C., Abreu, L., Baía, I., \& Samorinha, C. (2019). Concerns with educating the public about donating and receiving gametes. The European Journal of Contraception \& Reproductive Health Care, 24(6), 420-421. https://doi.org/10.1080/13625187.2019.1662392 Oosterhoff, B., \& Palmer, C. A. (2020). Attitudes and psychological factors associated with news monitoring, social distancing, disinfecting, and hoarding behaviors among US adolescents during the coronavirus disease 2019 pandemic. JAMA Pediatrics, E7-E7. https://doi.org/10.1001/jamapediatrics.2020.1876

República Portuguesa. (2020). Estamoson. Resposta de Portugal à COVID-19. Medidas excecionais. Retrieved from https://covid19estamoson.gov.pt/medidas-excecionais/

Rice, R. E., \& Atkin, C. K. (2013). Public communication campaigns. Sage.

Rosenstock, I. M. (1966). Why people use health services. Millbank Memorial Fund Quarterly, 44, 94-124. https://doi.org/10.1111/j.1468-0009.2005.00425.x 
Rosenstock, I. M. (2005). Why people use health services. Milbank Quarterly, 83, $p p-p p$. https://doi.org/10.1111/j.1468-0009.2005.00425.x

SNS - Serviço Nacional de Saúde. (2020). COVID-19. Retrieved from https://www.sns24.gov.pt/tema/doencas-infecciosas/covid-19/\#sec-0

Teti, M., Schatz, E., \& Liebenberg, L. (2020). Methods in the time of COVID-19: The vital role of qualitative inquiries. International Journal of Qualitative Methods, 19, 1-5. https://doi.org/10.1177/1609406920920962

WHO - World Health Organization. (2020a). Coronavirus disease (COVID-19). Weekly epidemiological update. Retrieved from https://www.who.int/docs/default-source/coronaviruse/situationreports/20200914-weekly-epi-update-5.pdf?sfvrsn=cf929d04_2

WHO - World Health Organization. (2020b). Coronavirus disease (COVID-19) pandemic. Retrieved from https://www.who.int/emergencies/diseases/novel-coronavirus-2019

Zhong, B.-L., Luo, W., Li, H.-M., Zhang, Q.-Q., Liu, X.-G., Li, W.-T., \& Li, Y. (2020). Knowledge, attitudes, and practices towards COVID-19 among Chinese residents during the rapid rise period of the COVID-19 outbreak: a quick online cross-sectional survey. International Journal of Biological Sciences, 16(10), 1745-1752. https://doi.org/10.7150/ijbs.45221

Historial do artigo

Recebido $\quad 06 / 2020$

Aceite $\quad 09 / 2020$

Publicado $12 / 2020$ 